\title{
ASPP2 Inhibits the Profibrotic Effects of Transforming Growth Factor- $\beta 1$ in Hepatic Stellate Cells by Reducing Autophagy
}

\author{
Minghua Lin ${ }^{1} \cdot$ Yuan Chang ${ }^{1} \cdot$ Fang Xie ${ }^{1} \cdot$ Ying Shi $^{1} \cdot$ Lijun Pang $^{1} \cdot$ Dexi Chen ${ }^{1,2}$
}

Received: 11 April 2016 / Accepted: 19 October 2017 / Published online: 2 December 2017

(c) The Author(s) 2017. This article is an open access publication

\begin{abstract}
Background Apoptosis-stimulating protein of p53-2 (ASPP2) is a damage-inducible P53-binding protein that enhances damage-induced apoptosis. Fibrosis is a wound-healing response, and hepatic stellate cells (HSCs) are key players in liver fibrogenesis. However, little is known about the relationship between ASPP2 and hepatic fibrosis.

Aims We investigated the effects of ASPP2 overexpression in HSCs and the role of ASPP2 in mouse liver fibrogenesis. Methods Human HSCs (LX-2 cells) were pre-incubated with GFP adenovirus (Ad) or ASPP2 adenovirus (AdASPP2) for $24 \mathrm{~h}$ and then treated with or without TGF- $\beta 1$. ASPP2 ${ }^{+/-}$and ASPP2 $+/+$Balb/c mice were used to examine the effects of ASPP2 on liver fibrosis in vivo. ASPP2+/+ Balb/c mice were generated by injecting AdASPP2 into the tail vein of ASPP2 WT Balb/c mice; all mice received intraperitoneal injections of carbon tetrachloride.

Results In this study, ASPP2 was found to markedly inhibit TGF- $\beta 1$-induced fibrogenic activation of LX- 2 cells. Further experiments using an autophagic flux assay confirmed that ASPP2 reduced the fibrogenic activation of LX-2 cells by inhibiting autophagy. Moreover, we found that ASPP 2 overexpression attenuated the anti-apoptotic effects of TGF- $\beta 1$ in LX- 2 cells. The extent of liver fibrosis was markedly reduced in ASPP2+/+ mouse liver tissue compared with control mice; however, in ASPP2 ${ }^{+/-}$mice, hepatic collagen deposition was significantly increased.

Conclusion These results suggest that TGF- $\beta 1$-induced autophagy is required for the fibrogenic response in LX- 2 cells and that ASPP2 may both inhibit TGF- $\beta 1$-induced autophagy and decrease liver fibrosis.
\end{abstract}

Keywords ASPP $2 \cdot$ Autophagy $\cdot$ Transforming growth factor- $\beta 1 \cdot$ Hepatic stellate cell

\section{Introduction}

Liver fibrosis, which is the excessive accumulation of extracellular matrix (ECM) proteins, severely increases the risk of developing liver failure and hepatocellular carcinoma [1].

Electronic supplementary material The online version of this article (doi:10.1007/s10620-017-4816-3) contains supplementary material, which is available to authorized users.

Minghua Lin and Yuan Chang have contributed equally to this work.

Dexi Chen

dexichen@ccmu.edu.cn

1 Beijing Youan Hospital, Beijing Institute of Hepatology, Capital Medical University, Beijing 100069, China

2 Organ Transplantation Center, The Affiliated Hospital of Qingdao University, Qingdao City 266003, Shandong Province, China
Hepatic stellate cells (HSCs) are liver-specific mesenchymal cells that play vital roles in liver physiology and fibrogenesis $[2,3]$. Transforming growth factor- $\beta 1$ (TGF- $\beta 1)$ is involved in the regulation of many cellular processes, including cell proliferation, differentiation, and inflammation $[4$, 5]. During chronic liver injury, the expression of TGF- $\beta 1$ significantly increases in both an autocrine and paracrine manner, thus activating hepatic stellate cells and resulting in increased secretion of ECM [6-8]. Autophagy is a critical lysosomal pathway that maintains cell function and survival through the degradation of cellular components [9-11]. Recently published data indicate that HSC activation is associated with autophagy, and studies have shown that blocking autophagy in HSCs attenuates liver fibrosis [12-14].

ASPP2, also known as 53BP2L, is encoded by TP53BP2 and is a member of a new family of proteins that includes the pro-apoptotic ASPP1 and anti-apoptotic iASPP [15-17]. Endogenous ASPP2 is damage-inducible and modulates physiologic damage response pathways involved in diverse 
cellular functions $[16,20]$. Numerous studies have confirmed that ASPP2 inhibits cell growth and stimulates apoptosis $[18,19]$. Moreover, low expression of ASPP2 is frequently found in a variety of human cancers, such as breast cancer, where low ASPP2 mRNA expression levels are associated with poor clinical outcomes [19, 21, 22]. However, the pathophysiologic role of ASPP2 in liver fibrogenesis has not been studied.

In this study, we explored a novel function of ASPP2 in the process of liver fibrogenesis. Understanding the pathophysiologic role of ASPP2 in this process will potentially provide new therapies that repress liver fibrosis.

\section{Materials and Methods}

\section{Cell Culture and Treatment}

Human HSCs (LX-2 cells) were grown in Dulbecco's modified Eagle's medium (DMEM) containing 10\% fetal bovine serum. After pre-incubation with GFP-adenovirus (Ad) or ASPP2 adenovirus (AdASPP2) at a concentration of $1 \times 10^{6}$ $\mathrm{PFU} / \mathrm{ml}$ for $24 \mathrm{~h}$, the LX-2 cells were treated with or without TGF- $\beta 1$ (10 ng/ml, PeproTech).

\section{Real-Time RT-PCR}

Total RNA was extracted from cultured LX-2 cells and liver tissue using an RNeasy kit (Qiagen) and reverse transcribed using the SuperScript ${ }^{\circledR}$ III First-Strand Synthesis System (Invitrogen). Real-time RT-PCR was performed in the ViiA 7DX sequence detecting system (Applied Biosystems). Relative transcript levels of target genes were normalized with $18 \mathrm{~S}$ rRNA levels. Primers were as follows: $18 \mathrm{~S}$ rRNA: sense, 5'-GTA ACC CGT TGA ACC CCA TT-3'; antisense, 5'-CCA TCC AAT CGG TAG TAG CG-3'. Human Col a1(I): sense, 5'-CCC CAC TCA GCC CAG TGT-3'; antisense, 5'-ACC AGA CAT GCC TCT TGT CCT T-3'. Human Col a1(III): sense, 5'-GGT TTT GCC CCG TAT TAT GGA3'; antisense, 5'-GAA GTC ATA ATC TCA TCG GTG TTG A-3'. Human a-SMA: sense, 5'-TGC CTG ATG GGC AAG TGA T-3'; antisense, 5'-GTC TCT GGG CAG CGG AAA3'. Human Bax: sense, 5'-AGT GGC AGC TGA CAT GT-3'; antisense, 5'-AGG GCC TTG AGC ACC AGT-3'. Human Bcl-2: sense, 5'-ATG GCG CAC GCT GGG AGA A-3'; antisense, 5'-ATG GAT GTA CTT CAG CAC TAT-3'. Mouse Col a1(I): sense, 5'-AGG GCG AGT GCT GTG CTT T-3'; antisense, 5'-CCC TCG ACT CCT ACA TCT TCT GA-3'. Mouse Col a1(III): sense, 5'-TGA AAC CCC AGC AAA ACA AAA-3'; antisense, 5'-TCA CTT GCA CTG GTT GAT AAG ATT AA- $3^{\prime}$. Mouse $\alpha$-SMA: sense, $5^{\prime}$-ATG CTC CCA GGG CTG TTT T-3'; antisense, 5'-TTC CAA CCA TTA CTC CCT GAT GT-3'. Mouse ASPP2: sense, 5'-AGC TGC
CAT GGA GAC CAT CT-3'; antisense, 5'-ACT GTT CTC CGT ACT GGC AC-3'.

\section{Western Blot Analysis}

Western blot analysis was performed using $50 \mu \mathrm{g}$ of protein extract obtained as described previously [15]. Antibodies against a-SMA, ASPP2, and LC3B were purchased from Sigma Aldrich; polyclonal antibodies against caspase 3 were purchased from Abcam. Peroxidase-conjugated goat anti-mouse or goat anti-rabbit IgG antibodies were used as secondary antibodies. Protein expression was visualized using an enhanced chemiluminescence (ECL Plus) assay kit according to the manufacturer's instructions. The results were normalized relative to the expression of glyceraldehyde-3-phosphate dehydrogenase (GAPDH).

\section{Immunofluorescence and Immunohistochemistry}

Liver samples were fixed in $4 \%$ paraformaldehyde and embedded in Tissue Tek OCT compound (Sakura Finetek USA, Inc.); 7- $\mu \mathrm{m}$ frozen sections were used for immunofluorescence. They were blocked with $2 \%$ bovine serum albumin for $1 \mathrm{~h}$ and then incubated with anti- $\alpha$-SMA monoclonal antibody (1:500 sigma) and anti-ASPP2 polyclonal antibody(1:500 invitrogen) followed by Alexa Fluor 488- or 546-conjugated secondary antibody (Sigma-Aldrich). The sections were covered with Vectashield mounting medium containing 4,6-diamidino-2-phenylindole and observed under a confocal microscope (Leica TCS SP8, Germany). For negative controls, sections were processed as described earlier, except that incubation with the primary antibody was omitted.

LX-2 cells were fixed with $4 \%$ paraformaldehyde and permeabilized with $0.5 \%$ Triton X-100 for $15 \mathrm{~min}$. Then, cells were blocked with $2 \%$ BSA for $1 \mathrm{~h}$ and incubated with anti-M30 monoclonal antibody (produced by our laboratory) and Alexa Fluor 546-conjugated secondary antibody. DAPI was used to stain the nuclei.

Immunohistochemical analysis with Masson stain was performed to detect hepatic collagen deposition.

\section{RNA Interference}

Small interfering RNA (siRNA) specific to human ASPP2 (target sequence: 5-AAGTTGCTGAGCAGGAGAAAC-3) and a nonspecific control were synthesized by Genepharmer. LX-2 cells of 40-50\% confluency were prepared for siRNA transfection. Transient transfection of siRNA $(35 \mathrm{nM})$ was performed using FuGENE HD transfection reagent (Promega, Madison, WI, USA). 


\section{Evaluation of Fluorescent LC3 Puncta}

LX-2 cells cultured on coverslips were infected with Ad-tfLC3 at 20 MOI. Twenty-four hours after adenovirus infection, the cells were washed with PBS, fixed with $4 \%$ paraformaldehyde, and viewed with a fluorescence microscope (Nikon Ti-E, Japan). The number of GFP and mRFP dots was determined by manual counting of fluorescent puncta in five fields from 30 different cells using a $40 \times$ objective. Each experiment was repeated three times. The number of dots per cell was calculated by dividing the total number of dots by the number of cells in each microscopic field.

\section{Mouse Models}

$\mathrm{ASPP}^{+/-}$Balb/c mice were kindly provided by Dr. Lopez et al. [16]. ASPP2 wild-type (WT) Balb/c mice (Academy of Military Medical Sciences, China), and ASPP2+/+ Balb/c mice (overexpressing ASPP2) were generated from ASPP2 WT Balb/c mice by injecting ASPP2-ad (60,000 viral particles/mice) into the tail vein. Control mice were generated from ASPP2 WT by injecting Control-ad (60,000 viral particles/mice) into the tail vein. All mice received intraperitoneal injections of a carbon tetrachloride $\left(\mathrm{CCl}_{4}\right)$ /olive oil $(\mathrm{OO})$ mixture $(1: 9 \mathrm{v} / \mathrm{v})$ twice a week $(1 \mu \mathrm{l}$ per gram body weight). Mice were killed after 6 weeks of $\mathrm{CCl}_{4}$ treatment, and tissues were harvested the day after the final administration of $\mathrm{CCl}_{4}$. All studies were approved by the Ethics Committee of the Youan Hospital Center and the hospital affiliated with Capital Medical University, Beijing. All animal care complied with applicable health guidelines.

\section{Statistical Analysis}

The results are expressed as the mean \pm SEM. Statistical significance was determined using the Student's $t$ test. A $p$ value $<0.05$ was considered significant.

\section{Results}

\section{ASPP2 Reduces TGF- $\beta 1$-Induced Fibrogenic Activation of LX-2 Cells}

The activation of HSCs plays a pivotal role in liver fibrogenesis [1]. TGF- $\beta 1$ is the classic fibrogenic cytokine involved in accelerating the progression of liver fibrosis [6]. Therefore, it was of interest to investigate the effects of ASPP2 on the TGF- $\beta 1$-induced fibrogenic activation of LX-2 cells. First, LX-2 cells were pre-treated with ASPP2adenovirus (AdASPP2) or GFP-adenovirus (Ad) for $24 \mathrm{~h}$ and then treated with or without TGF- $\beta 1$ ( $10 \mathrm{ng} / \mathrm{ml})$. Next, to investigate the role of ASPP2 in the fibrogenic activation of
LX-2 cells, we examined the expression of fibrotic markers [ $\alpha$-SMA, Col $\alpha 1$ (I), and Col $\alpha 1$ (III)]. Quantitative analysis showed that the mRNA expression of $\alpha$-SMA, Col $\alpha 1$ (I), and Col $\alpha 1$ (III) was significantly upregulated in TGF$\beta 1$ and Ad-treated cells compared to cells treated with Ad alone (Fig. 1A). However, the enhanced mRNA expression of $\alpha$-SMA, Col $\alpha 1(\mathrm{I})$, and Col $\alpha 1$ (III) mediated by TGF$\beta 1$ was blunted by pre-incubation with AdASPP2. Likewise, TGF- $\beta 1$-mediated increases in $\alpha$-SMA protein levels were inhibited by AdASPP2 pre-incubation in LX-2 cells (Fig. 1B, C). We also observed that AdASPP2 treatment did not affect the viability of LX-2 cells (data not shown). In addition, we found that ASPP2 overexpression in LX-2 cells not only affects TGF- $\beta 1$-induced fibrogenic activation, but also attenuates basal fibrogenic activation. To test this issue, siRNA technology was used to knock down ASPP2 expression, as shown in Fig. 1D. Infection of LX-2 cells with ASPP2 siRNA markedly increased the expression of $\alpha$-SMA induced by TGF- $\beta 1$ treatment. Taken together, these results suggest that ASPP2 may reduce the TGF- $\beta 1$-induced fibrogenic activation of LX-2 cells.

To further confirm these results, we analyzed the changes in ASPP2 gene and protein expression during the activation of primary cultured mouse HSCs. Culture-activated HSCs expressed 2.3 times as much ASPP2 mRNA as quiescent HSCs, and ASPP2 protein levels were also clearly increased in culture-activated HSCs. (Supplementary Figure 1).

\section{ASPP2 Inhibits the Profibrotic Effects of TGF- $\beta 1$ by Reducing Autophagy in LX-2 Cells}

Recent studies have shown that TGF- $\beta 1$-induced autophagy is required for the fibrogenic response in human atrial myofibroblasts (hATMyofbs) [17] and that ASPP2 can inhibit autophagy through a p53-independent pathway [18]. Therefore, we speculated whether ASPP2 could decrease TGF- $\beta 1$-induced profibrotic effects in LX- 2 cells by inhibiting autophagy. Western blot analysis showed that $\alpha$-SMA and LC3-II protein levels were markedly elevated in Ad and TGF- $\beta 1$-treated cells, and this upregulation was abolished by AdASPP2 treatment (Fig. 2A). The graphical representation of this Western blotting data, including statistical analysis, is shown in Fig. 2B. To determine whether the ASPP2-mediated decrease of fibrogenic activation in LX-2 cells is associated with ASPP2-mediated inhibition of autophagy, we cotreated LX-2 cells with 3-methyladenine (3MA, an inhibitor of autophagy) and TGF- $\beta 1$ for $24 \mathrm{~h}$, and $\alpha$-SMA and LC3-II expression was examined. The results were similar to those observed when cells were treated with both AdASPP2 and TGF- $\beta 1$, as shown in Fig. $2 \mathrm{~A}$, B. These results support the hypothesis that ASPP2 decreases TGF- $\beta 1$-induced profibrotic effects in LX-2 cells through inhibiting autophagy. To further confirm this hypothesis, we detected autophagic 


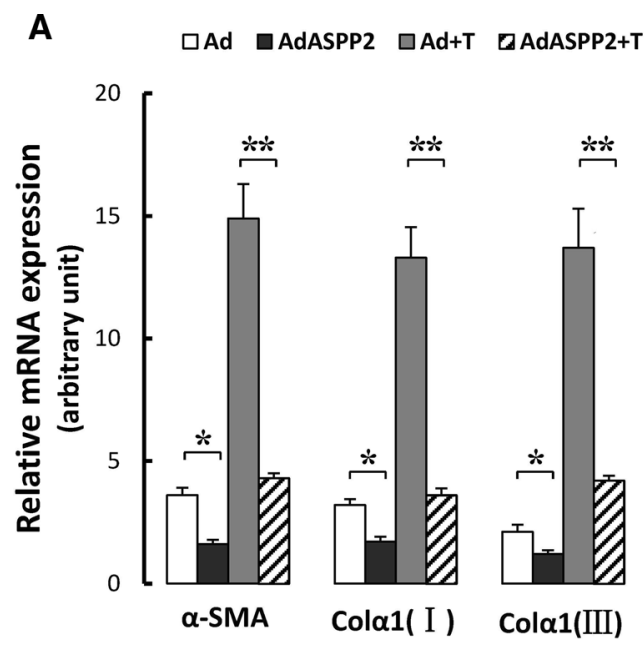

B
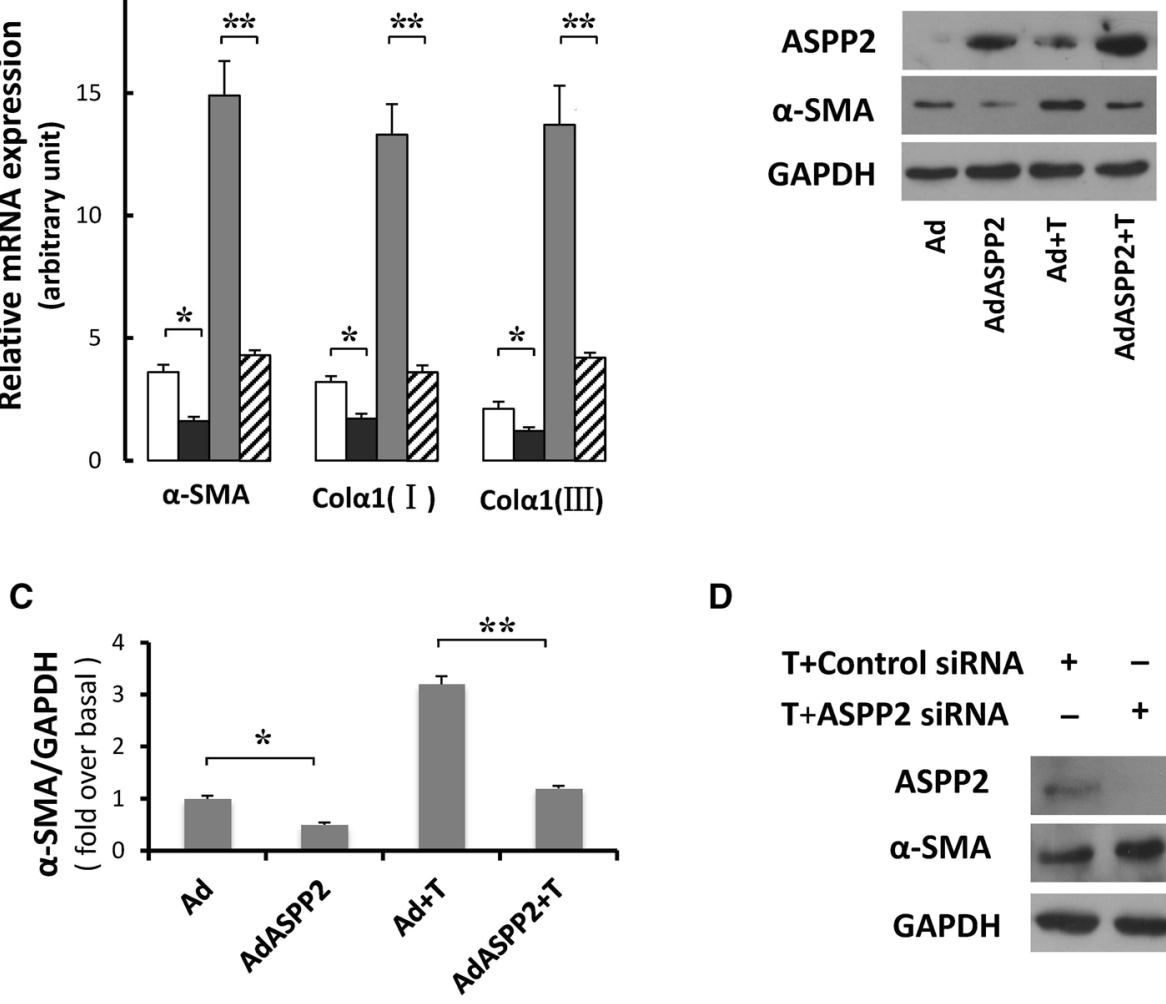

D

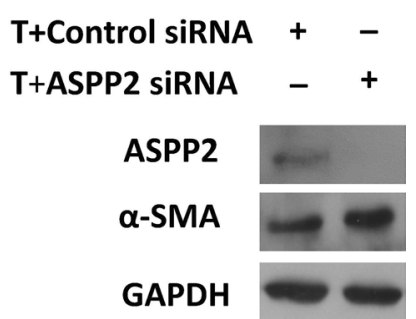

Fig. 1 ASPP2 reduces TGF- $\beta 1$-induced fibrogenic activation of LX-2 cells. LX-2 cells were pre-treated with Ad (GFP-adenovirus) or AdASPP2 (ASPP2-adenovirus) for $24 \mathrm{~h}$ and then treated with or without TGF- $\beta 1(10 \mathrm{ng} / \mathrm{ml})$ for $12 \mathrm{~h}$. a The mRNA expression of $\alpha$-SMA, Cola1(I), and Cola1(III) was measured by real-time RT-PCR. Cells were treated with Ad, AdASPP2, Ad and TGF- $\beta 1$, AdASPP2, and TGF- $\beta 1$, respectively. b Western blot analysis of the $\alpha$-SMA and ASPP2 protein level. $\mathbf{c}$ Typical autoradiograms are

flux by using adenovirus harboring tf-LC3 (Ad-tf-LC3) [19, 24]. Next, the number of autophagosomes can be evaluated by determining the number of red dots that overlay green dots and appear yellow in merged images. The red dots that do not overlay green dots and appear red in merged images indicate autolysosome formation. As shown in Fig. 2C, D, the numbers of green and red dots per cell were both significantly decreased after treatment with AdASPP2 and TGF- $\beta 1$ compared to Ad and TGF- $\beta 1$ treatment. These results suggested that ASPP2 may reduce the fibrogenic activation of LX- 2 cells by inhibiting TGF- $\beta 1$-induced autophagy.

\section{ASPP2 Inhibits Anti-apoptotic Effects of TGF- $\beta 1$ in LX-2 Cells}

It has been shown that ASPP2 may induce tumor cell apoptosis [9]. Selective stimulation of apoptosis in stellate cells (rather than hepatocytes) may have anti-fibrotic shown. After quantification of the signals, results were normalized relative to GAPDH expression. d Effect of silencing ASPP2 expression on the fibrogenic activation of LX-2 cells in response to TGF$\beta 1$. Lane 1 was cells treated with control siRNA and TGF- $\beta 1$; lane 2 was cells treated with ASPP2 siRNA and TGF- $\beta 1$. Results represent the means from at least three independent experiments. Data are presented as the mean \pm SEM. ${ }^{*} p<0.05$ versus Ad, $* * p<0.05$ versus $\mathrm{Ad}+\mathrm{T}$

effects [20-23]. Thus, we questioned whether ASPP2 might induce apoptosis in HSCs and reduce liver fibrosis. First, we detected apoptosis by performing M30 staining, and we observed that the number of M30-positive cells was markedly increased in cells treated with AdASPP2 and TGF- $\beta 1$ compared with Ad and TGF- $\beta 1$-treated cells (Fig. 3A, B). Quantitative analysis of the expression of apoptosis-related genes Bax and Bcl-2 was performed by qRT-PCR. There was an approximately 2.5 -fold increase in Bax mRNA (Fig. 3C) and a threefold decrease in Bcl-2 mRNA (Fig. 3D) in cells treated with AdASPP2 and TGF- $\beta 1$ compared with Ad and TGF- $\beta 1$-treated cells. To confirm this finding, we further analyzed apoptosis levels using immunoblotting. As shown in Fig. 3E, cleaved caspase 3 fragment levels were markedly increased after ASPP2 overexpression. These data support the idea that ASPP2 may inhibit the anti-apoptotic effects of TGF- $\beta 1$ in LX-2 cells. 




Fig. 2 ASPP2 inhibits the profibrotic effect of TGF- $\beta 1$ by reducing autophagy in LX-2 cells. a Expression of ASPP2, $\alpha$-SMA, and LC3 was determined by Western blot after 24-h treatment with ASPP2 adenovirus or 3-MA. Lanes 1 and 2 are cells treated with Ad or AdASPP2 without treatment with TGF- $\beta 1$; lanes 3,4 , and 5 are cells treated with Ad, AdASPP2, and 3MA followed by TGF- $\beta 1$. b Typical autoradiograms are shown. After quantification of the signals, results were normalized relative to GAPDH expression. c Autophagy

\section{ASPP2 Attenuates $\mathrm{CCl}_{4}$-Induced Liver Fibrosis in Mice}

Finally, we analyzed the effect of ASPP2 on mouse liver fibrosis induced by $\mathrm{CCl}_{4}$. We used mice with low ASPP2 expression (ASPP2 ${ }^{+-}$) [28] and mice overexpressing ASPP2 (ASPP2+/+), which were obtained by injecting AdASPP2 into the tail vein of ASPP2 WT mice. ASPP2 mRNA levels were significantly lower in $\mathrm{ASPP}^{+/-}$mice compared with ASPP2 WT mice after 6 weeks of $\mathrm{CCl}_{4}$ treatment; meanwhile, ASPP2 mRNA levels were significantly higher in ASPP $2+/+$ mice compared with Ad-treated mice (Fig. 4A). Likewise, Western blot analysis revealed a similar change in ASPP2 protein levels (Fig. 4B). In addition, we found that ASPP2 is predominantly expressed in hepatocytes and HSCs during $\mathrm{CCl}_{4}$-induced liver fibrosis (Fig. 4C). Next, we evaluated the effect of ASPP2 on hepatic collagen deposition with morphometric analysis of Masson staining and quantified any changes with digital image analysis. As shown in Fig. 4D, E, collagen deposition was significantly increased
B

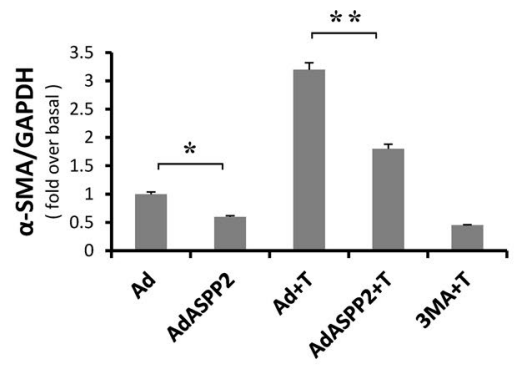

D

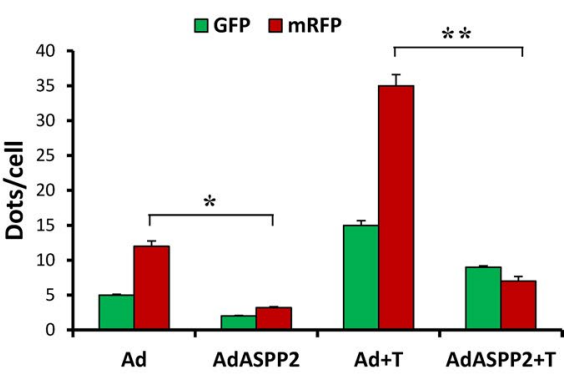

flux detection was performed in LX-2 cells infected by mRFP-GFPLC3 adenovirus and ASPP2-ad/Control-ad and then treated with or without TGF- $\beta 1$ ( $\times 400$ magnification). d Mean number of GFP and mRFP dots per cell. mRFP-GFP-LC3 adenovirus was infected at 20 MOI. Results represent the means from at least three independent experiments. Data are presented as the mean \pm SEM. ${ }^{*} p<0.05$ versus $\mathrm{Ad}, * * p<0.05$ versus $\mathrm{Ad}+\mathrm{T}$

in ASPP2 $2^{+/-}$mice compared with ASPP2 WT mice after 6 weeks of $\mathrm{CCl}_{4}$ administration (Fig. 4D-a, b, E). In contrast, collagen deposition was markedly decreased in ASPP $2+/+$ mouse liver tissues compared with control mouse liver tissues (Fig. 4D-c, d, E). We also examined the effect of ASPP2 on the expression of fibrosis markers [ $\alpha$-SMA, Col $\alpha 1$ (I) and Col $\alpha 1$ (III)] in mouse liver tissues. As shown in Fig. 4E, the levels of $\alpha$-SMA, Col $\alpha 1$ (I), and Col $\alpha 1$ (III) mRNA were significantly increased in liver tissues of ASPP2 ${ }^{+/-}$mice compared with WT mice. However, compared with control mice, ASPP2 overexpression (ASPP2+/+) resulted in a marked reduction in $\alpha$-SMA, Col $\alpha 1$ (I), and Col $\alpha 1$ (III) mRNA levels.

To identify the effects of ASPP2 on autophagy in liver tissue of $\mathrm{CCL}_{4}$-treated mice, we performed an immunofluorescence assay to detect LC3 specks in fibrotic mouse liver tissue. LC3 specks were detected in most of the cells from $\mathrm{ASPP}^{+/-}$mice, but few were detected in ASPP2+/+ mice (Supplementary Figure 2). Finally, analysis of biochemical parameters (alanine aminotransferase, ALT) demonstrated that ASPP2 affected the extent of inflammation and necrosis 
in the liver. These results indicate that ASPP2 attenuates $\mathrm{CCl}_{4}$-induced mouse liver fibrosis.

\section{Discussion}

Liver fibrosis, characterized by an accumulation of extracellular matrix, is the final common symptom of chronic liver disease [1,24]. Experimental and clinical reports published in the last decade have suggested that both the removal of etiology as well as effective therapy can result in significant regression of liver fibrosis, particularly in animal models [25-32]. However, the mechanisms of this regression have not been fully clarified.

Autophagy is a critical lysosomal pathway that maintains cell function and survival through the degradation of cellular components. The liver depends on autophagy to both maintain normal function and prevent the development of disease states. However, Hernández-Gea et al. [13] suggest that autophagy promotes the development of hepatic fibrosis. Our results also support this conclusion. TGF- $\beta 1$ is an important profibrotic factor that markedly increases during
Fig. 4 The effect of ASPP2 on $\mathrm{CCl}_{4}$-induced liver fibrosis in mice. ASPP2 $+/+$ mice and CON-Ad mice were obtained by injecting ASPP2-Ad or CON-Ad into the tail vein of ASPP2 wild-type (WT) $\mathrm{BALB} / \mathrm{c}$ mice ( $n=6$ per group). All the mice were treated with $\mathrm{CCL}_{4}$ through intraperitoneal injection for 6 weeks. Control mice received olive oil (OO) treatment. a Relative hepatic level of ASPP2 mRNA was measured by real-time RT-PCR. b Western blot analysis of ASPP2 protein level was shown in mouse liver tissue. c Colocalization of $\alpha$-SMA and ASPP 2 by double immunostaining, Sections are stained for $\alpha$-SMA (red) and ASPP2 (green) followed by confocal microscopy. DAPI is used to visualize nuclei (blue). The inset indicates negative control staining ( $\times 400$ magnification). d Masson's trichrome staining $(\times 100$ magnification). Inset: Masson staining for OO-treated livers. e Quantitative analysis of liver fibrosis, ten randomly selected fields were quantitated for each mouse using the NIS-Elements BR software. f Expression of $\alpha$-SMA, Col $\alpha 1(\mathrm{I})$, and Col $\alpha 1(\mathrm{III}) \mathrm{mRNA}$ in mouse liver tissue. $\mathrm{g}$ Serum aminotransferase ALT was measured using commercial assay kits. ALT, alanine aminotransferase. Data are expressed as the mean \pm SEM. ${ }^{*} p<0.05$ versus WT mice, ${ }^{* *} p<0.05$ versus CON-Ad mice

liver fibrosis, ultimately causing the acceleration of liver fibrosis. Our in vitro experiments revealed that TGF- $\beta 1$ may significantly increase the fibrogenic activation of LX-2 cells by promoting autophagy. These results are consistent with
A

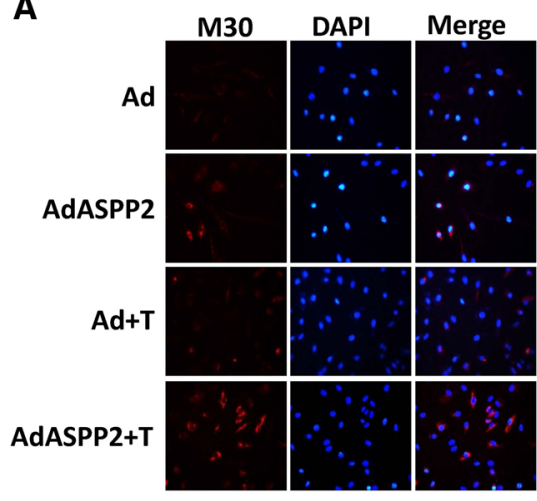

B

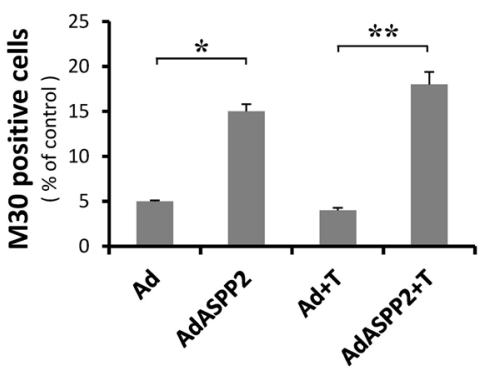

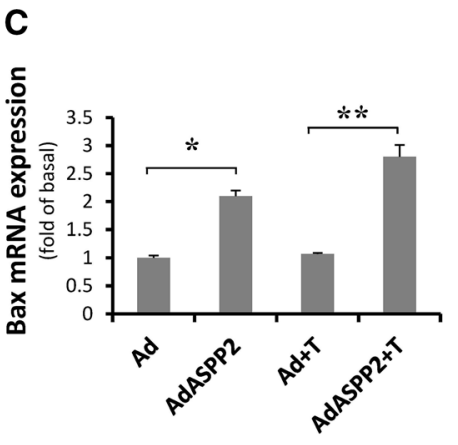

C

D

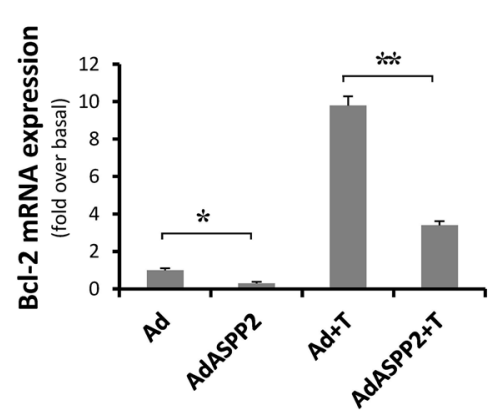

E

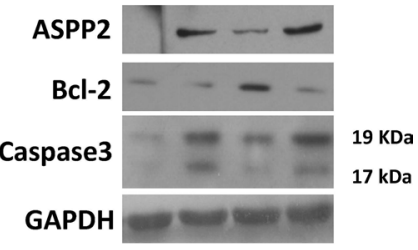



Fig. 3 ASPP2 inhibits the anti-apoptotic effect of TGF- $\beta 1$ in LX-2 cells. a Detection of apoptosis was performed by immunostaining of M30 (× 200 magnification). b The percentage of M30-positive cells was quantified by analyzing at least ten random fields per slice. The expression of Bax (c) and Bcl-2 (d) mRNA, measured by real- time RT-PCR. e Western blot analyses of Bcl-2 and activated caspase 3 expression were performed. Results represent the means from at least three independent experiments. Data are presented as the mean \pm SEM. $* p<0.05$ versus $\mathrm{Ad},{ }^{*} * p<0.05$ versus $\mathrm{Ad}+\mathrm{T}$ 

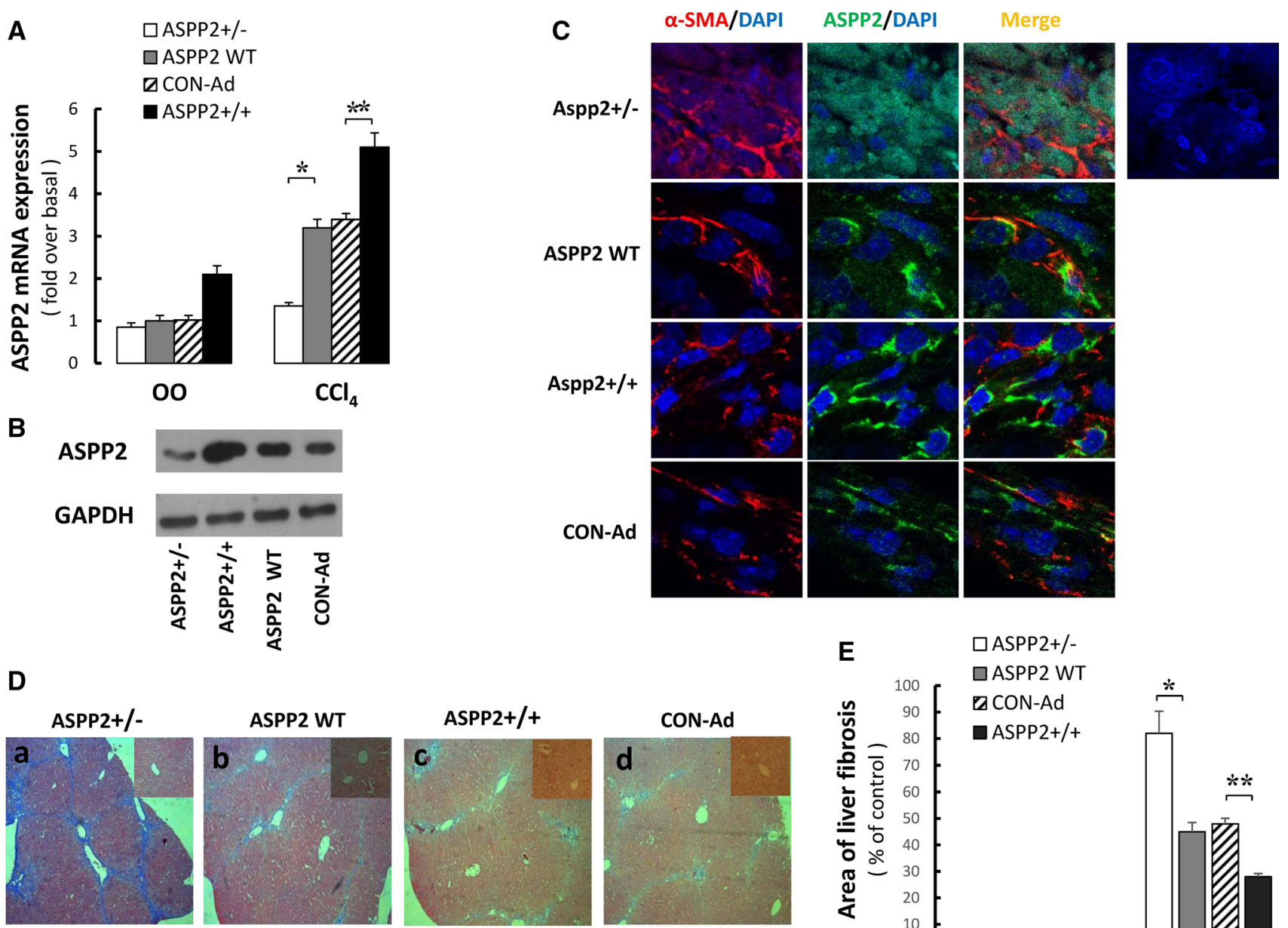

ASPP2 WT
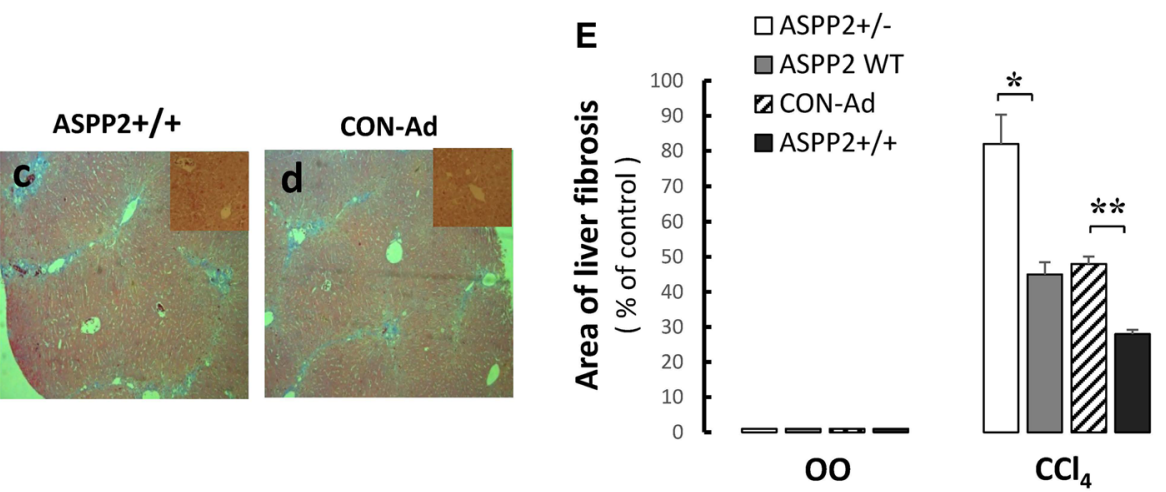

$\mathbf{F}$

$\square$ ASPP2+/- $\square$ ASPP2 WT $\square$ CON-Ad $\square$ ASPP2+/+

G
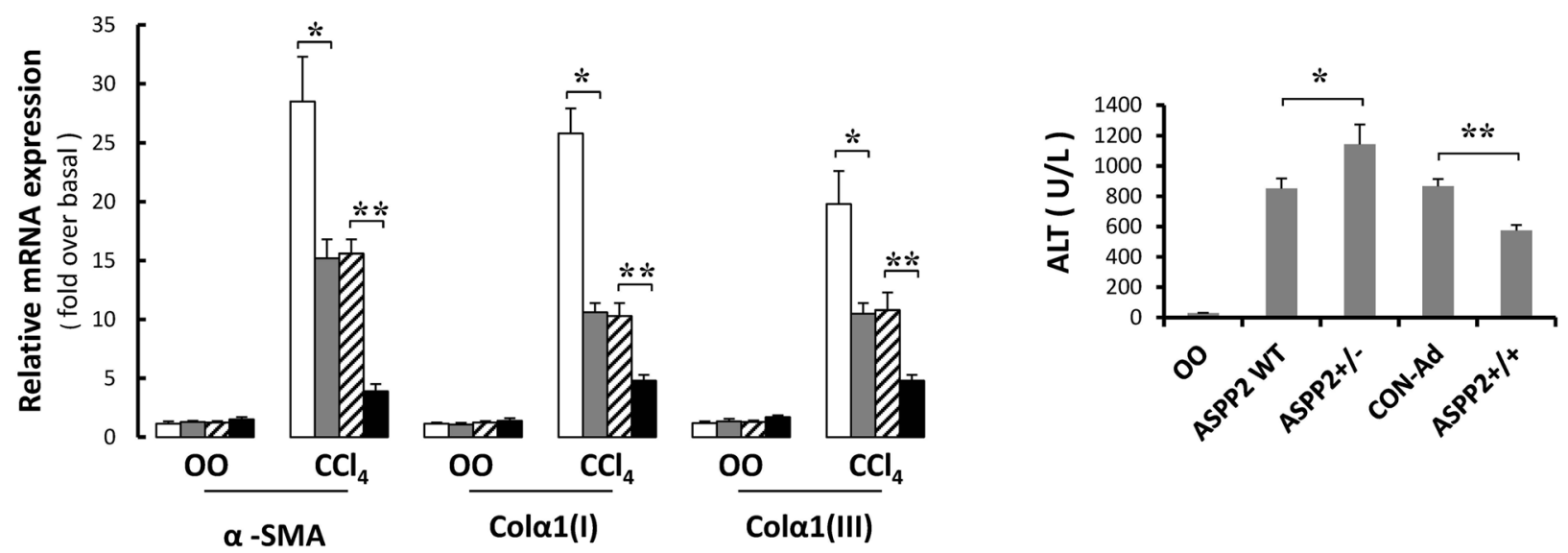

studies by Ghavami et al. [17]. The suppression of autophagy in hepatic stellate cells may be a novel antifibrotic strategy.

ASPP2 is a tumor suppressor that stimulates P53-mediated apoptosis [33, 34]. Recently, ASPP2 has been reported to regulate autophagy. Wang et al. [18] suggest that ASPP2 may inhibit autophagy. Conversely, Liu et al. [35] show that ASPP2 may induce autophagy. The reason for such discrepancies is not clear, although one explanation may be that these studies utilized different cell types. Our previous findings indicated that ASPP2 mRNA 
expression is upregulated after 7 days of $\mathrm{CCL}_{4}$ administration. After 7 days, these increases continue in a way that is negatively correlated with the progression of liver fibrosis. Maximal ASPP2 mRNA levels are achieved after 6 weeks of $\mathrm{CCL}_{4}$ treatment. Therefore, we questioned whether ASPP2 may reduce liver fibrosis by inhibiting autophagy in HSCs. In this study, we showed that TGF- $\beta 1$ may significantly increase the fibrogenic activation of LX-2 cells by promoting their autophagy. However, using adenovirus technology, we demonstrated that ASPP2 overexpression markedly reduces the TGF- $\beta 1$-induced fibrogenic activation of LX-2 cells, leading to decreased expression of fibrogenic genes $\alpha$-SMA, Col $\alpha 1(\mathrm{I})$, and Col $\alpha 1(\mathrm{III})$. More importantly, our data indicate that ASPP2 overexpression may inhibit TGF- $\beta 1$-induced autophagy in LX-2 cells. In addition, our studies also show that ASPP2 overexpression inhibits the anti-apoptotic effects of TGF- $\beta 1$ in LX- 2 cells.

In vivo, we observed a relationship between ASPP2 levels and the degree of liver fibrosis using ASPP2 $2^{+/}$, ASPP2 WT, CON-Ad, and ASPP2+/+ mice treated with $\mathrm{CCL}_{4}$. We observed that collagen deposition was significantly increased in ASPP2 ${ }^{+/-}$mice compared with ASPP2 WT mice after 6 weeks of $\mathrm{CCl}_{4}$ administration. In contrast, collagen deposition was markedly decreased in ASPP2+/+ mouse liver tissues compared with control mouse liver tissues. Lopez et al. demonstrated that endogenous ASPP2 is damage-inducible and that a biologic function of endogenous ASPP2 is to modulate physiologic damage response pathways [16]. In our fibrotic mouse model, the administration of $\mathrm{CCL}_{4}$ led to mouse liver injury and the upregulation of ASPP2. However, it was unclear whether the beneficial effects of ASPP2 were from HSCs or hepatocytes. We isolated the mouse primary hepatocytes, but the experiment could not be completed for various reasons. Therefore, we used HepG2 cells induced by LPS; the results indicated that ASPP2 overexpression may effectively prevent the LPS damage to HepG2 cells and reduce apoptosis (data not shown). The above results are consistent with our previous article-ASPP2 attenuates triglycerides to protect against hepatocyte injury by reducing autophagy in a cell and mouse model of nonalcoholic fatty liver disease [36]. Here, we only want to investigate the role of ASPP2 overexpression in TGF-induced hepatic stellate cell activation, with hepatic stellate cells as target cells. Taken together, these data suggest that TGF- $\beta 1$-induced autophagy is required for the fibrogenic response in LX-2 cells, whereas ASPP2 may inhibit TGF$\beta 1$-induced autophagy and decrease liver fibrosis.

In conclusion, this study describes an additional function for ASPP2: it participates in the wound-healing response in the liver. We provide new clues to the molecular mechanisms underlying liver fibrosis regression, which may reveal new perspectives for the pharmacologic treatment of liver fibrosis.

Acknowledgments This study was financially supported by the National Natural and Science Foundation of China (81672026); Basics-Clinical Research Cooperation Fund of Capital Medical University, China (15JL-L05); Beijing Municipal Institute of Public Medical Research Development and Reform pilot project (JING YI YAN 2016-2); Beijing Precision Medicine and Transformation Engineering Technology Research Center of Hepatitis and Liver Cancer.

\section{Compliance with ethical standards}

Conflict of interest All authors declare that they have no competing financial interests related to this work.

Open Access This article is distributed under the terms of the Creative Commons Attribution-NonCommercial 4.0 International License (http://creativecommons.org/licenses/by-nc/4.0/), which permits any noncommercial use, distribution, and reproduction in any medium, provided you give appropriate credit to the original author(s) and the source, provide a link to the Creative Commons license, and indicate if changes were made.

\section{References}

1. Friedman SL. Mechanisms of hepatic fibrogenesis. Gastroenterology. 2008;134:1655-1669.

2. Yin C, Evason KJ, Asahina K, et al. Hepatic stellate cells in liver development, regeneration, and cancer. J Clin Invest. 2013; 123:1902-1910.

3. Friedman SL. Hepatic stellate cells: protean, multifunctional, and enigmatic cells of the liver. Physiol Rev. 2008;88:125-172.

4. Kajdaniuk D, Marek B, Borgiel-Marek H, et al. Transforming growth factor beta1 (TGFbeta1) in physiology and pathology. Endokrynol Pol. 2013;64:384-396.

5. Blobe GC, Schiemann WP, Lodish HF. Role of transforming growth factor beta in human disease. $N$ Engl J Med. 2000;342:1350-1358.

6. Inagaki Y, Okazaki I. Emerging insights into Transforming growth factor beta Smad signal in hepatic fibrogenesis. Gut. 2007;56:284-292.

7. Breitkopf K, Godoy P, Ciuclan L, et al. TGF-beta/Smad signaling in the injured liver. Z Gastroenterol. 2006;44:57-66.

8. Leask A, Abraham DJ. TGF-beta signaling and the fibrotic response. FASEB J. 2004;18:816-827.

9. Czaja MJ, Ding WX, Donohue TM, Jr, et al. Functions of autophagy in normal and diseased liver. Autophagy. 2013;9:1131-1158.

10. Levine B, Klionsky DJ. Development by self-digestion: molecular mechanisms and biological functions of autophagy. Dev Cell. 2004;6:463-477.

11. He C, Klionsky DJ. Regulation mechanisms and signaling pathways of autophagy. Annu Rev Genet. 2009;43:67-93.

12. Thoen LF, Guimarães EL, Dollé L, et al. A role for autophagy during hepatic stellate cell activation. $J$ Hepatol. 2011;55:1353-1360.

13. Hernández-Gea V, Ghiassi-Nejad Z, Rozenfeld R, et al. Autophagy releases lipid that promotes fibrogenesis by activated hepatic stellate cells in mice and in human tissues. Gastroenterology. 2012;142:938-946. 
14. Thoen LF, Guimaraes EL, Grunsven LA. Autophagy: a new player in hepatic stellate cell activation. Autophagy. 2012;8:126-128.

15. Shi Y, Han Y, Xie F, et al. ASPP2 enhances oxaliplatin (L-OHP)induced colorectal cancer cell apoptosis in a p53-independent manner by inhibiting cell autophagy. J Cell Mol Med. 2015;19:535-543.

16. Lopez CD, Ao Y, Rohde LH, et al. Proapoptotic p53-interacting protein 53BP2 is induced by UV irradiation but suppressed by p53. Mol Cell Biol. 2000;20:8018-8025.

17. Ghavami S, Cunnington RH, Gupta S, et al. Autophagy is a regulator of TGF-beta1-induced fibrogenesis in primary human atrial myofibroblasts. Cell Death Dis. 2015;6:e1696.

18. Wang Y, Wang XD, Lapi E, et al. Autophagic activity dictates the cellular response to oncogenic RAS. Proc Natl Acad Sci USA. 2012;109:13325-13330.

19. Rubinsztein DC, Cuervo AM, Ravikumar B, et al. In search of an "autophagomometer". Autophagy. 2009;5:585-589.

20. Iredale JP. Hepatic stellate cell behavior during resolution of liver injury. Semin Liver Dis. 2001;21:427-436.

21. Iredale JP, Benyon RC, Pickering J, et al. Mechanisms of spontaneous resolution of rat liver fibrosis. Hepatic stellate cell apoptosis and reduced hepatic expression of metalloproteinase inhibitors. $J$ Clin Invest. 1998;102:538-549.

22. Wright MC, Issa R, Smart DE, et al. Gliotoxin stimulates the apoptosis of human and rat hepatic stellate cells and enhances the resolution of liver fibrosis in rats. Gastroenterology. 2001;121:685-698.

23. Oakley F, Meso M, Iredale JP, et al. Inhibition of inhibitor of kappaB kinases stimulates hepatic stellate cell apoptosis and accelerated recovery from rat liver fibrosis. Gastroenterology. 2005;128:108-120.

24. Friedman SL. Liver fibrosis-from bench to bedside. J Hepatol. 2003;38:S38-S53.

25. Povero D, Busletta C, Novo E, et al. Liver fibrosis: a dynamic and potentially reversible process. Histol Histopathol. 2010;25:1075-1091.

26. Issa $\mathrm{R}$, Zhou $\mathrm{X}$, Constandinou $\mathrm{CM}$, et al. Spontaneous recovery from micronodular cirrhosis: evidence for incomplete resolution associated with matrix cross-linking. Gastroenterology. 2004;126:1795-1808.

27. Tugues S, Fernandez-Varo G, Muñoz-Luque J, et al. Antiangiogenic treatment with sunitinib ameliorates inflammatory infiltrate, fibrosis, and portal pressure in cirrhotic rats. Hepatology. 2007;46:1919-1926.

28. Julien B, Grenard P, Teixeira-Clerc F, et al. Antifibrogenic role of the cannabinoid receptor CB2 in the liver. Gastroenterology. 2005; 128:742-755.

29. Dienstag JL, Goldin RD, Heathcote EJ, et al. Histological outcome during long-term lamivudine therapy. Gastroenterology. 2003; 124:105-117.

30. Poynard T, McHutchison J, Manns M, et al. Impact of pegylated interferon alfa-2b and ribavirin on liver fibrosis in patients with chronic hepatitis C. Gastroenterology. 2002;122:1303-1313.

31. Murphy FR, Issa R, Zhou X, et al. Inhibition of apoptosis of activated hepatic stellate cells by tissue inhibitor of metalloproteinase- 1 is mediated via effects on matrix metalloproteinase inhibition-implications for reversibility of liver fibrosis. J Biol Chem. 2002;277:11069-11076.

32. Hammel P, Couvelard A, O'Toole D, et al. Regression of liver fibrosis after biliary drainage in patients with chronic pancreatitis and stenosis of the common bile duct. $N$ Engl J Med. 2001;344:418-423.

33. Tordella L, Koch S, Salter V, et al. ASPP2 suppresses squamous cell carcinoma via RelA/p65-mediated repression of p63. Proc Natl Acad Sci USA. 2013;110:17969-17974.

34. Kampa KM, Acoba JD, Chen D, et al. Apoptosis-stimulating protein of p53 (ASPP2) heterozygous mice are tumor-prone and have attenuated cellular damage-response thresholds. Proc Natl Acad Sci USA. 2009;106:4390-4395.

35. Liu K, Shi Y, Guo X, et al. CHOP mediates ASPP2-induced autophagic apoptosis in hepatoma cells by releasing Beclin-1 from $\mathrm{Bcl}-2$ and inducing nuclear translocation of Bcl-2. Cell Death Dis. 2014;5:e1323.

36. Xie F, Jia L, Lin M, et al. ASPP2 attenuates triglycerides to protect against hepatocyte injury reducing autophagy in a cell and mouse model of non-alcoholic fatty liver disease. J Cell Mol Med. 2015;19:155-164. 